\title{
A universal planar graph under the minor relation
}

\author{
Reinhard Diestel Daniela Kühn
}

\begin{abstract}
We construct an infinite planar graph that contains every planar graph as a minor.
\end{abstract}

\section{Introduction}

Answering a question of Ulam, Pach [7] proved that there is no 'universal planar graph', i.e. no planar graph that contains every other planar graph as a subgraph. The purpose of this paper is to show that the answer becomes positive if we replace the subgraph relation by the (weaker) minor relation: we shall construct a planar graph $G^{*}$ that contains every other planar graph as a minor. See [5] for a survey of universality results based on the subgraph relation.

Following a short section on terminology, the construction of $G^{*}$ is presented in Section 3. Its universality is then proved in two stages. In Section 4 we show that every planar graph $G$ is the minor of some planar graph $H$ of maximum degree at most three. In Section 5 we show how any such $H$ may be embedded in $G^{*}$ as a minor. In Section 6 finally, we mention a few open problems about minor-universality.

\section{Terminology}

Our basic notation follows [1]. All the graphs in this paper are countable, and we assume that the edges of plane graphs are polygonal arcs. We impose no restrictions on graph drawings in terms of accumulation points. (Thus, a sequence of points from a plane graph $G$ may converge to any point of the plane, either on or off $G$.) Universal graphs for such restricted drawings are considered in [6].

Minors for infinite graphs are defined exactly as for finite graphs (see [1]). Note that their branch sets, the connected vertex sets to be contracted, may be infinite. 
We further assume a fixed orientation of $\mathbb{R}^{2}$. If $C$ is a cycle embedded in $\mathbb{R}^{2}$, we denote by $\vec{C}$ (respectively $\overleftarrow{C}$ ) the cycle $C$ oriented in the direction agreeing with (respectively opposite) this orientation of $\mathbb{R}^{2}$. We shall also call this direction clockwise (respectively anticlockwise). Hence, if $f$ is a face in a plane graph, and $\vec{e}$ is an oriented edge on the boundary of $f$, then $f$ lies on the right of $\vec{e}$ or on its left (or both) in a natural way. (Think of 'clockwise' as a right turn.) For two vertices $v, w$ on $C$ we write $v \vec{C} w$ for the subpath of $C$ from $v$ to $w$ following the clockwise orientation of $C$. The path $v \overleftarrow{C} w$ is defined correspondingly.

Whenever $v_{1} \cdots v_{n} v_{1}$ is a cycle, we put $v_{n+1}:=v_{1}$. The inner face of a plane cycle $C$ will be denoted by $f(C)$. A path in $C \cup f(C)$ that avoids $C$ except possibly in its first and last vertex is said to run through $f(C)$.

\section{Construction of $G^{*}$}

Since every finite planar graph is a minor of some large enough finite grid, a first candidate for $G^{*}$ might be the infinite grid. Unfortunately this does not work: the graph obtained from $K^{4}$ by joining to each of its four vertices infinitely many new vertices of degree one is planar but not a minor of the infinite grid. Our plan is to construct $G^{*}$ inductively, accommodating at each step all the possible ways in which a plane graph to be embedded in $G^{*}$ as a minor might unfold vertex by vertex.

Let a cycle of type $n$ denote a plane cycle of length $2^{n+3}$ whose vertices are coloured red and blue alternately. We shall construct an infinite sequence $G_{0}^{*} \subseteq G_{1}^{*} \subseteq \ldots$ of finite plane graphs and, for each $G_{n}^{*}$, a set $\mathcal{C}_{n}$ of disjoint cycles of type $n$ in $G_{n}^{*}$ each bounding an inner face of $G_{n}^{*}$.

Let $G_{0}^{*}$ be a drawing of the 8-cycle $C^{8}$, colour its vertices red and blue alternately, and let $\mathcal{C}_{0}$ consist of the cycle $G_{0}^{*}$. Now suppose we have constructed $G_{0}^{*}, \ldots, G_{n}^{*}$ and $\mathcal{C}_{0}, \ldots, \mathcal{C}_{n}$ as desired. In the inner face of each cycle $C=: u_{1} \cdots u_{2^{n+3}} u_{1}$ in $\mathcal{C}_{n}$ insert a cycle $C^{\prime}$ of type $n+1$ and $2^{n+3}$ disjoint paths of length $2^{n+2}-1$ linking the vertices of $C$ to the blue vertices of $C^{\prime}$. Denote the path linking a vertex $v$ on $C$ to $C^{\prime}$ by $P_{v}$ and the vertex of $P_{v}$ on $C^{\prime}$ by $v^{\prime}$ (Fig. 1 ).

Let $C_{i}:=u_{i} u_{i+1} \cup P_{u_{i+1}} \cup u_{i}^{\prime} \vec{C}^{\prime} u_{i+1}^{\prime} \cup P_{u_{i}}$. Each of these cycles $C_{i}$ bounds an inner face in the plane graph thus obtained. Insert a new cycle $C_{i}^{\prime}$ of type $n+1$ in this face, and join its blue vertices bijectively to the vertices of $P_{u_{i}} \cup P_{u_{i+1}}$, to obtain another plane graph (Fig. 2). Let this graph be $G_{n+1}^{*}$.

For each cycle $C \in \mathcal{C}_{n}$, we call $C^{\prime}$ the large cycle of $G_{n+1}^{*}$ inside $C$ and 


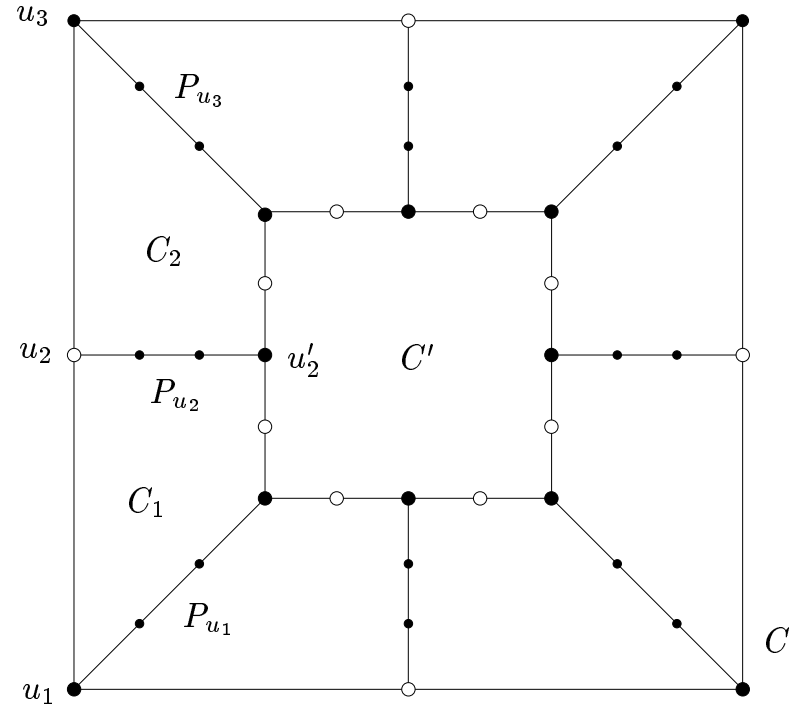

Figure 1: Adding $C^{\prime}$ for $n=0$

each $C_{i}^{\prime}$ the small cycle of $G_{n+1}^{*}$ inside $C$ preceding $u_{i+1}$. Setting

$$
\begin{aligned}
\mathcal{C}_{n+1}: & =\left\{C^{\prime} \mid C \in \mathcal{C}_{n}\right\} \cup\left\{C_{i}^{\prime} \mid C \in \mathcal{C}_{n} ; i=1, \ldots, 2^{n+3}\right\} \\
& =\left\{D \mid \exists C \in \mathcal{C}_{n}: D \text { is the large cycle or a small cycle of } G_{n+1}^{*} \text { inside } C\right\}
\end{aligned}
$$

then completes the induction step of our construction.

Finally, we define the plane graph $G^{*}$ by setting $G^{*}:=\bigcup_{n=0}^{\infty} G_{n}^{*}$.

Let us fix some more notation. Let $C_{0}$ be a cycle in $\mathcal{C}_{n}, v_{0}$ a vertex on $C_{0}, k \geq 1$ and $C_{k}$ a cycle in $\mathcal{C}_{n+k}$. We say that $C_{k}$ is the large cycle of $G_{n+k}^{*}$ inside $C_{0}$ if there exist $C_{i} \in \mathcal{C}_{n+i}(i=1, \ldots, k-1)$ such that each $C_{i}$ is the large cycle of $G_{n+i}^{*}$ inside $C_{i-1}(i=1, \ldots, k)$. In this case $G^{*}$ contains a unique path $P_{v_{0}}^{k}$ from $v_{0}$ to $C_{k}$ that is the union of $C_{i-1}-C_{i}$ paths of the form $P_{v}$. Indeed, let $P_{v_{0}}^{0}:=v_{0}$ and inductively define $P_{v_{0}}^{i+1}:=P_{v_{0}}^{i} \cup P_{v_{i}}$, where $v_{i}$ is the vertex of $P_{v_{0}}^{i}$ on $C_{i}$ and $0 \leq i \leq k-1$.

For distinct vertices $v, w$ on some cycle $C \in \mathcal{C}_{n}$ we put $C_{v w}:=$ $v \vec{C} w P_{w} w^{\prime} \overleftarrow{C}^{\prime} v^{\prime} P_{v} v$, where $C^{\prime}$ (as in the construction of $G_{n+1}^{*}$ ) is the large cycle of $G_{n+1}^{*}$ inside $C$. 


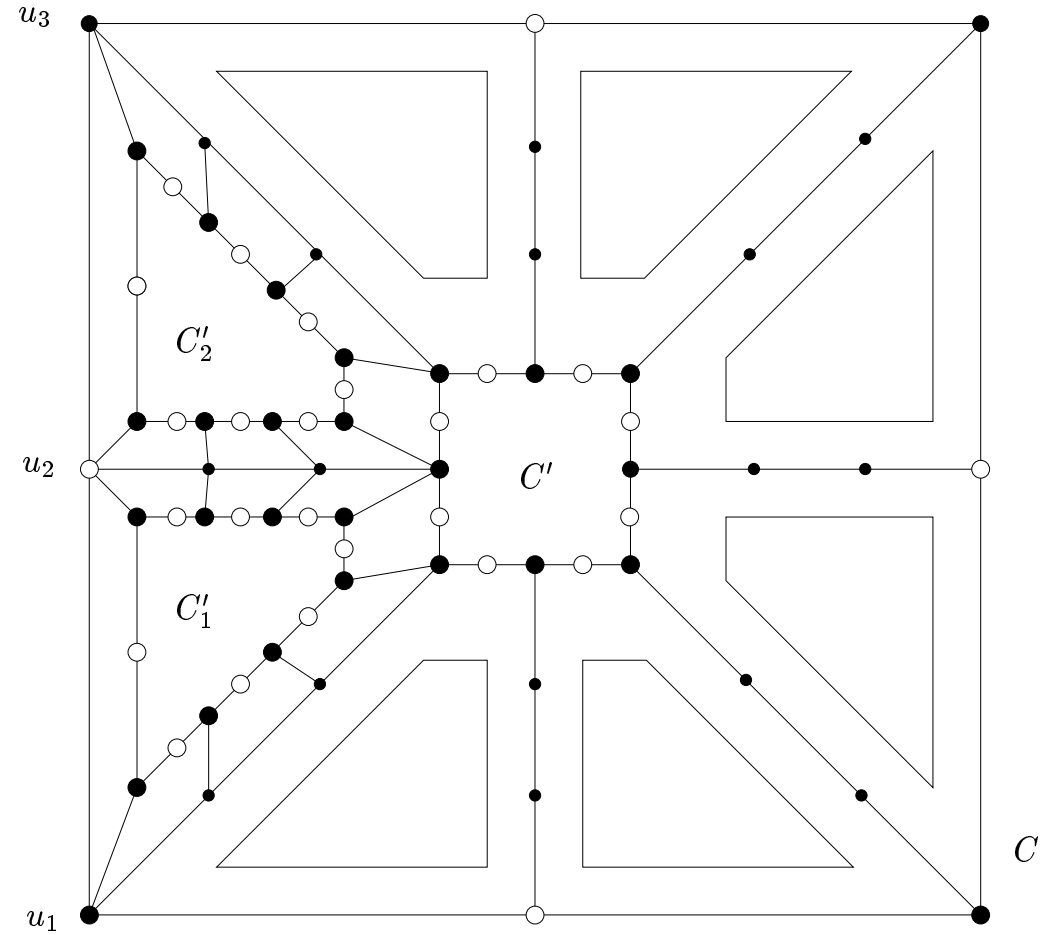

Figure 2: Inserting the cycles $C_{i}^{\prime}$ for $n=0$ to form $G_{1}^{*}$

Lemma 1 Let $C \in \mathcal{C}_{n}, k \geq 0$ and let $v_{k}, \ldots, v_{0}, w_{0}, \ldots, w_{k}$ be distinct blue vertices on $C$ in clockwise order. Then $G_{n+k}^{*}$ contains disjoint paths $P_{0}, \ldots, P_{k}$ such that $P_{i}$ joins $v_{i}$ to $w_{i}(i=0, \ldots, k), P_{0}$ is a subpath of $C$, and $P_{1}, \ldots, P_{k}$ run through $f(C)$.

Proof. Let $C_{0}:=C$, and for $i=1, \ldots, k$ let $C_{i}$ be the large cycle of $G_{n+i}^{*}$ inside $C_{0}$. For $i=0, \ldots, k$ let $P_{i}$ be the unique $v_{i}-w_{i}$ path in $P_{v_{i}}^{i} \cup C_{i} \cup P_{w_{i}}^{i}$ whose segment in $C_{i}$ follows the clockwise orientation of $C_{i}$ (Fig. 3).

Lemma 2 Let $v, w$ be distinct vertices on $C \in \mathcal{C}_{n}$. Let $P_{v}=: v_{0} \cdots v_{2^{n+2}-1}$ and $P_{w}=: w_{0} \cdots w_{2^{n+2}-1}$ where $v_{0}=v$ and $w_{0}=w$. Let $k \leq 2^{n+2}-2$. Then $G_{n+k}^{*}$ contains disjoint paths $P_{1}, \ldots, P_{k}$ running through $f\left(C_{v w}\right)$, such that for all $i=1, \ldots, k$ :

(i) $P_{i}$ joins $v_{i}$ to $w_{i}$; 
The idea is to replace each vertex of $G$ by a tree of maximum degree at most three, and to join up the leaves of these trees according to the edges of $G$, retaining planarity. This cannot be done arbitrarily, since we may inadvertently create $K_{3,3}$ minors in this way (Fig. 4). In our proof of the
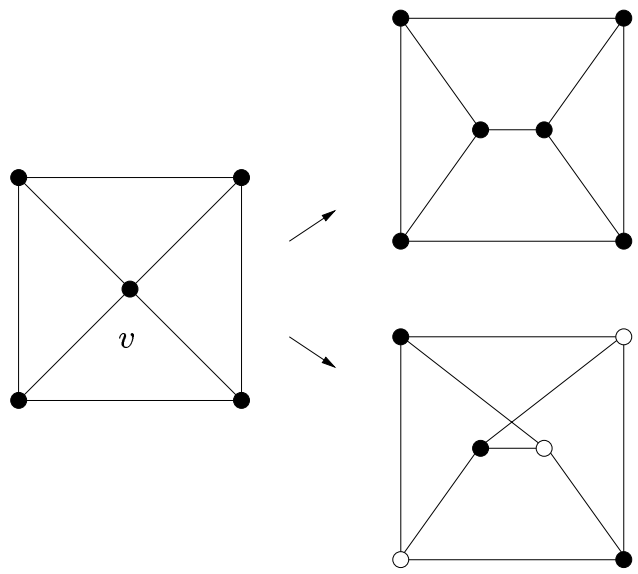

Figure 4: A planar and a non-planar expansion of the vertex $v$

lemma, we shall therefore construct $H$ as a plane graph, designed with reference to a fixed drawing of $G$. Thus we assume in the following that $G$ is a plane graph.

We start with some definitions. Let $X$ be a finite plane graph and $v$ a vertex of $X$, and let $e_{1}, \ldots, e_{k}$ be the edges incident with $v$ (in clockwise order). Let $e_{k+1}:=e_{1}$. For all $i=2, \ldots, k+1$ we call $e_{i}$ the successor of $e_{i-1}$ at $v$ and $e_{i-1}$ the predecessor of $e_{i}$ at $v$. (If $k=1$, then $e_{1}$ is its own successor and predecessor.)

Let $T$ be a plane tree with distinct leaves $v_{1}, v_{2}, v_{3}$. Let $P=x_{1} \cdots x_{n}$ be the $v_{1}-v_{2}$ path in $T$ (so $v_{1}=x_{1}$ and $v_{2}=x_{n}$ ), and let $P^{\prime}=y_{1} \cdots y_{m}$ be the $v_{3}-P$ path in $T$, with $y_{m}=x_{l}$ say. Thus $1<l<n$. If $x_{l} x_{l+1}$ is the successor of $y_{m-1} y_{m}$ at $x_{l}=y_{m}$ in the plane graph $P \cup P^{\prime}$, we say that $v_{3}$ lies between $v_{1}$ and $v_{2}$ in $T$; note that the order of $v_{1}$ and $v_{2}$ matters here. For distinct leaves $v_{1} \neq v_{2}$ of $T$, we also say that $v_{2}$ lies between $v_{1}$ and $v_{1}$.

Proof of Lemma 3. We may assume that $G$ is connected and has at least two vertices. Indeed, if $G$ is not connected, then adding a new vertex to $G$ and joining it to one vertex in every component of $G$ does not create a $K^{5}$ or $K_{3,3}$ minor. Thus, by the infinite version of Kuratowski's theorem the 
graph obtained is planar, and we may consider this graph instead of $G$.

Let us fix an infinite sequence $\left(G_{n}\right)_{n=0}^{\infty}$ of finite connected plane subgraphs of $G$ such that

(i) if $G_{n}=G$ then $G_{n+1}=G$; if not, then $G_{n+1}$ is obtained from $G_{n}$ either by adding a new edge or by adding a new vertex and joining it to a vertex of $G_{n}$;

(ii) $G=\bigcup_{n=0}^{\infty} G_{n}$;

(iii) $\left|G_{0}\right|=2$.

We shall inductively construct finite connected plane graphs $H_{0} \subseteq H_{1} \subseteq \ldots$ together with partitions $\left\{V_{x}^{n} \mid x \in V\left(G_{n}\right)\right\}$ of $V\left(H_{n}\right)$, satisfying the following conditions for all $n \in \mathbb{N}$ :

(a) $V_{x}^{n} \subseteq V_{x}^{n+1}$ for all $x \in G_{n}$.

(b) For all $x \in G_{n}$ the subgraph $T_{x}^{n}$ of $H_{n}$ induced by $V_{x}^{n}$ is a tree of maximum degree at most three, whose leaves have degree at most two in $H_{n}$.

(c) For distinct $x, y \in G_{n}$, there is a $V_{x}^{n}-V_{y}^{n}$ edge in $H_{n}$ if and only if $x y$ is an edge of $G_{n}$. This $V_{x}^{n}-V_{y}^{n}$ edge is unique, and its end $v_{x y}$ in $V_{x}^{n}$ is a leaf of $T_{x}^{n}$.

(d) If $x_{1}, x_{2}$ are neighbours of $x$ in $G_{n}$ (not necessarily distinct), then $T_{x}^{n}$ has a leaf between $v_{x x_{1}}$ and $v_{x x_{2}}$.

(e) If $x, x_{1}, x_{2} \in G_{n}$ and $x x_{2}$ is the successor of $x x_{1}$ at $x$ in $G_{n}\left(x_{1}\right.$ and $x_{2}$ may coincide), then every leaf of $T_{x}^{n}$ between $v_{x x_{1}}$ and $v_{x x_{2}}$ has degree one in $H_{n}$.

(f) There is a bijection $\varphi_{n}$ between the faces of $G_{n}$ and those of $H_{n}$, with the following property: if $f$ is a face of $G_{n}$ lying on the left of an edge $\overrightarrow{x y}$ of $G_{n}$, then $\varphi_{n}(f)$ lies on the left of $\overrightarrow{v_{x y} v_{y x}}$ in $H_{n}$.

Clearly, the graph

$$
H:=\bigcup_{n=0}^{\infty} H_{n}
$$

will have maximum degree at most three and contain $G$ as a minor (with branch sets $V_{x}:=\bigcup_{n=0}^{\infty} V_{x}^{n}$ for all $\left.x \in G\right)$. 
Denote the vertices of $G_{0}$ by $x_{1}$ and $x_{2}$. Let $H_{0}$ be a drawing of the path $v_{1} v_{2} v_{3} v_{4}$, and let $V_{x_{1}}^{0}:=\left\{v_{1}, v_{2}\right\}$ and $V_{x_{2}}^{0}:=\left\{v_{3}, v_{4}\right\}$. Let $\varphi_{0}$ be the obvious bijection.

Now suppose we have defined $H_{0}, \ldots, H_{n}$ and $\left\{V_{x}^{i} \mid x \in G_{i}\right\}$ for all $i=$ $0, \ldots, n$, satisfying (a)-(f). We may assume that $G \neq G_{n}$, for if not let $H_{m}:=H_{n}, V_{x}^{m}:=V_{x}^{n}$ and $\varphi_{m}:=\varphi_{n}$ for all $m \geq n$ and all vertices $x \in G$.

In the construction of $H_{n+1}$ we will make use of the following property of $H^{n}$. Suppose $f$ is a face of $G_{n}, x x_{2}$ is the successor of $x x_{1}$ at $x$ in $G_{n}$, and $f$ lies on the left of $\overrightarrow{x_{1} \vec{x}}\left(x_{1}\right.$ and $x_{2}$ may coincide). Let $v$ be a leaf of $T_{x}^{n}$ between $v_{x x_{1}}$ and $v_{x x_{2}}$ (which exists by (d)). Then (c),(e) and (f) imply that $v$ has degree one in $H_{n}$ and lies on the boundary of $\varphi_{n}(f)$ (Fig. 5).
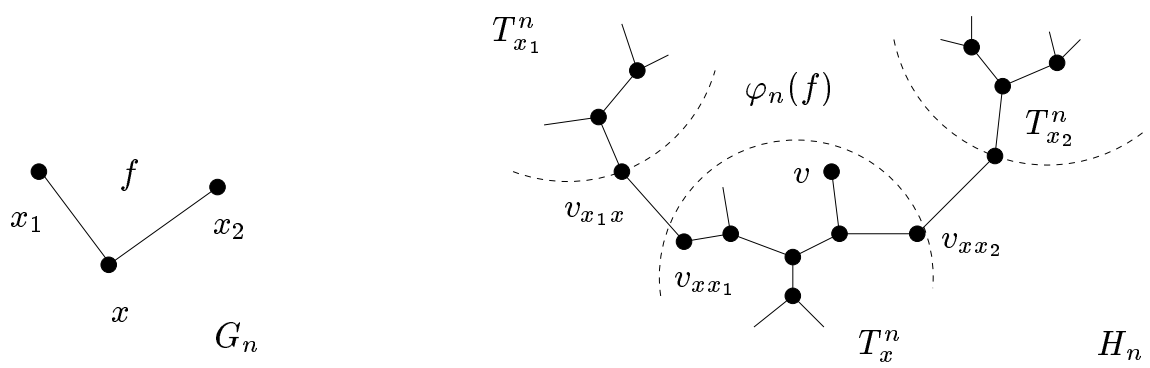

Figure 5: Expanding $G_{n}$ to $H_{n}$

Case 1: $G_{n+1}$ was obtained from $G_{n}$ by adding an edge $e=x y$ with $x, y \in G_{n}$.

Then there exist faces $f$ of $G_{n}$ and $f_{1}, f_{2}$ of $G_{n+1}$ such that $f=f_{1} \cup f_{2} \cup \stackrel{\circ}{e}$ and $f_{1}$ lies on the left of $\overrightarrow{x y}$ in $G_{n+1}$. (Note that $f_{1} \neq f_{2}$, since $G_{n}$ is connected.) Let $x x_{1}, y y_{1}$ be the predecessors of $x y$ in $G_{n+1}$ at $x$ and $y$, respectively, and let $x x_{2}, y y_{2}$ be the successors of $x y$ in $G_{n+1}$ at $x$ and $y$, respectively. By (d) we can find leaves $v_{x} \in T_{x}^{n}$ and $v_{y} \in T_{y}^{n}$ such that $v_{x}$ lies between $v_{x x_{1}}$ and $v_{x x_{2}}$, and $v_{y}$ lies between $v_{y y_{1}}$ and $v_{y y_{2}}$ Observe that $f$ lies on the left of both $\overrightarrow{x_{1} \vec{x}}$ and $\overrightarrow{y_{1} \vec{y}}$. Thus, as noted above, $v_{x}$ and $v_{y}$ both have degree one in $H_{n}$ and lie on the boundary of $\varphi_{n}(f)$. Join $v_{x}$ to $v_{y}$ inside $\varphi_{n}(f)$ by a path $P=v_{x} u_{1} \cdots u_{6} v_{y}$, so as to obtain another plane graph $H_{n}^{\prime}$. Since $H_{n}$ is connected, $H_{n}^{\prime}$ has two faces $f_{1}^{\prime}$ and $f_{2}^{\prime}$ such that $f_{1}^{\prime} \cup f_{2}^{\prime} \cup \stackrel{\circ}{P}=\varphi_{n}(f)$

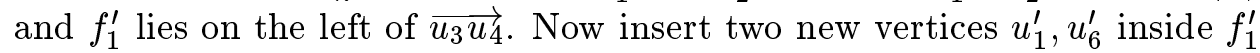
and two new vertices $u_{2}^{\prime}, u_{5}^{\prime}$ inside $f_{2}^{\prime}$. For $i=1,2,5,6$ join $u_{i}^{\prime}$ to $u_{i}$ so as to obtain another plane graph $H_{n+1}$ (Fig. 6). 
for the face $f_{1}$, since $\varphi_{n+1}\left(f_{1}\right)$ lies on the left of $\overrightarrow{u_{3} \overrightarrow{u_{4}}}=\overrightarrow{v_{x y} v_{y x}}$ by definition.

Case 2: $G_{n+1}$ was obtained from $G_{n}$ by adding a new vertex $x$ and joining it to some vertex $y \in G_{n}$.

Then there exist faces $f$ of $G_{n}$ and $f_{1}$ of $G_{n+1}$ such that $x y \backslash\{y\} \subseteq f$ and $f_{1}=f \backslash x y$. Let $y y_{1}$ be the predecessor and $y y_{2}$ the successor of $x y$ in $G_{n+1}$ at $y$. By (d) we can find a leaf $v$ of $T_{y}^{n}$ between $v_{y y_{1}}$ and $v_{y y_{2}}$. As before, $v$ has degree one in $H_{n}$ and lies on the boundary of $\varphi_{n}(f)$. Insert a new vertex $u$ inside $\varphi_{n}(f)$ and join it to $v$ by a path $P=u u_{1} u_{2} u_{3} u_{4} v$ inside $\varphi_{n}(f)$. Now insert two new vertices $u_{3}^{\prime}, u_{4}^{\prime}$ inside $\varphi_{n}(f) \backslash P$, and for $i=3,4$ join $u_{i}^{\prime}$ to $u_{i}$, to obtain another plane graph $H_{n+1}$ in which $u_{3} u_{3}^{\prime}$ is the successor of $u_{2} u_{3}$ at $u_{3}$ while $u_{4}^{\prime} u_{4}$ is the predecessor of $u_{4} u_{3}$ at $u_{4}$ (Fig. 7).

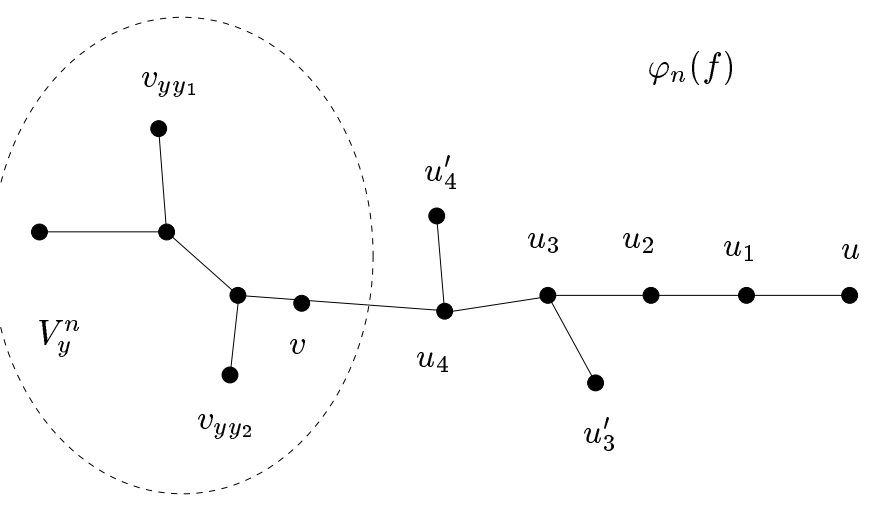

Figure 7: Extending $H_{n}$ to $H_{n+1}$ in Case 2

For all vertices $z \in G_{n+1}$ let

$$
V_{z}^{n+1}:= \begin{cases}\left\{u, u_{1}\right\} & \text { if } z=x \\ V_{y}^{n} \cup\left\{u_{2}, u_{3}, u_{4}, u_{3}^{\prime}, u_{4}^{\prime}\right\} & \text { if } z=y \\ V_{z}^{n} & \text { otherwise }\end{cases}
$$

Once more all faces $g \neq f_{1}$ of $G_{n+1}$ are also faces of $G_{n}$, and we let $\varphi_{n+1}(g):=\varphi_{n}(g)$. Finally, we let $\varphi_{n+1}\left(f_{1}\right)$ be the unique face of $H_{n+1}$ inside $\varphi_{n}(f)$.

This time all the conditions (a)-(f) are readily checked. 


\section{Universality of $G^{*}$}

Theorem 4 Every countable planar graph is a minor of $G^{*}$.

Proof. We show that every countably infinite connected plane graph $H$ of maximum degree at most three is a minor of $G^{*}$. The theorem then follows by Lemma 3. Subdividing an edge if $H$ is cubic, we may assume that $H$ has a vertex $x_{0}$ of degree two.

Note that there exists a sequence $\left(H^{n}\right)_{n=0}^{\infty}$ of finite connected subgraphs of $H$ such that:

(i) $H^{0}=\left\{x_{0}\right\}$;

(ii) $H^{n+1}$ is obtained from $H^{n}$ either by adding a new vertex $a$ and joining it to some vertex of $H^{n}$ or by adding a new edge between two vertices of $H^{n}$;

(iii) $H^{n+1}$ is obtained from $H^{n}$ by adding a new edge between two vertices $x, y \in H^{n}$ only if neither $x$ nor $y$ has a neighbour in $H-H^{n}$;

(iv) $H=\bigcup_{n=0}^{\infty} H^{n}$.

Our plan is to construct the branch set $V_{x} \subseteq V\left(G^{*}\right)$ of each vertex $x \in H$ inductively along the construction of $G^{*}$, as follows. For all $x \in H$ and all $n \in \mathbb{N}$ we shall define connected vertex sets $V_{x}^{n} \subseteq V\left(G^{*}\right)$ such that:

(a) $V_{x}^{n} \subseteq V_{x}^{n+1}$;

(b) $V_{x}^{n} \neq \emptyset$ if and only if $x \in H^{n}, V_{x}^{n} \cap V_{y}^{n}=\emptyset$ if $x \neq y$, and for every edge $x y$ in $H^{n}$ there is a $V_{x}^{n}-V_{y}^{n}$ edge in $G^{*}$;

(c) there exists $m(n) \geq n$ such that

(c1) $V_{x}^{n} \subseteq V\left(G_{m(n)}^{*}\right)$ for every $x \in H^{n}$;

(c2) for each vertex $x \in H^{n}$, the set $V_{x}^{n}$ meets a cycle in $\mathcal{C}_{m(n)}$ if and only if $d_{H^{n}}(x)<d_{H}(x)$; moreover, if $d_{H^{n}}(x)<d_{H}(x)$ then $V_{x}^{n}$ meets exactly one cycle $C_{x}^{n} \in \mathcal{C}_{m(n)}$, and it does so in a blue singleton $v_{x}^{n}$;

(d) if $P=x \cdots y$ is a nontrivial $H^{n}$-path in $H$ (i.e. $x, y$ are the only vertices of $P$ in $H^{n}$ and no edge of $P$ lies in $H^{n}$ ) then $V_{x}^{n}$ and $V_{y}^{n}$ meet the same cycle of $\mathcal{C}_{m(n)}$, i.e. $C_{x}^{n}=C_{y}^{n}$; 
(e) if $x_{1} \cdots y_{1}$ and $x_{2} \cdots y_{2}$ are disjoint nontrivial $H^{n}$-paths in $H$ with $C_{x_{1}}^{n}=C_{x_{2}}^{n}=: C$, then $v_{x_{2}}^{n}$ and $v_{y_{2}}^{n}$ lie in the same component of $C-\left\{v_{x_{1}}^{n}, v_{y_{1}}^{n}\right\}$.

(If $v_{x_{2}}^{n}$ and $v_{y_{2}}^{n}$ lie in different components of $C-\left\{v_{x_{1}}^{n}, v_{y_{1}}^{n}\right\}$, we say that $x_{1} \cdots y_{1}$ and $x_{2} \cdots y_{2}$ cross. Thus, (e) says that there are no crossing $H^{n}$-paths.)

Let $V_{x_{0}}^{0}$ consist of any blue vertex $v_{x_{0}}^{0}$ on $G_{0}^{*}$, and for all vertices $x \in H-H^{0}$ let $V_{x}^{0}:=\emptyset$. Then (a)-(e) hold with $m(0)=0$. Now let $n \geq 0$, and suppose we have defined $V_{x}^{i}$ for all vertices $x \in H$ and all $i=0, \ldots, n$.

The following two claims will be used to establish condition (e) in the induction step for the case that $H^{n+1}$ was obtained from $H^{n}$ by adding a new vertex $a$ and joining it to some vertex $x$ of $H^{n}$ (Case 1 below). Suppose that $x$ has another neighbour $b \neq a$ in $H$ (which may or may not lie in $H^{n}$ ), such that $x b$ is not an edge of $H^{n}$. Since $x$ has degree at most three in $H$ (degree two if $x=x_{0}$ ), and $x$ has at least one neighbour in $H^{n}$ (unless $n=0)$, any $\operatorname{such} b$ is unique.

Claim 1. Suppose that $b \in H^{n}$. Let $\mathcal{P}$ be the set of $H^{n}$-paths $P$ in $H$ that start with the edge $x a$. Let $z(P)$ denote the last vertex of $P$. Then either $v_{z(P)}^{n}$ lies in $v_{b}^{n} \vec{C}_{x}^{n} v_{x}^{n}$ for all paths $P \in \mathcal{P}$ (and we say that a belongs between $b$ and $x$ on $C_{x}^{n}$ ) or $v_{z(P)}^{n}$ lies in $v_{x}^{n} \vec{C}_{x}^{n} v_{b}^{n}$ for all paths $P \in \mathcal{P}$ (and we say that $a$ belongs between $x$ and $b$ on $C_{x}^{n}$ ).

Proof of Claim 1. Suppose the claim is false. Then we can find $P, P^{\prime} \in \mathcal{P}$ such that $\left\{v_{b}^{n}, v_{x}^{n}\right\}$ separates $v_{z(P)}^{n}$ from $v_{z\left(P^{\prime}\right)}^{n}$ in $C_{x}^{n}$. Then $P \cup P^{\prime}$ contains an $H^{n}$-path $z(P) \cdots z\left(P^{\prime}\right)$ that crosses $x b$, contradicting (e).

Claim 2. Suppose that $b \in H-H^{n}$. Let $\mathcal{P}$ be the set of pairs $\left(P_{1}, P_{2}\right)$ of $H^{n}$-paths in $H$ of the form $P_{1}=x a \cdots z\left(P_{1}\right)$ and $P_{2}=x b \cdots z\left(P_{2}\right)$, and such that $P_{1}$ and $P_{2}$ meet only in $x$. Then either $v_{z\left(P_{1}\right)}^{n}$ lies in $v_{z\left(P_{2}\right)}^{n} \vec{C}_{x}^{n} v_{x}^{n}$ for every pair $\left(P_{1}, P_{2}\right) \in \mathcal{P}$ (and we say that $a$ belongs between $b$ and $x$ on $\left.C_{x}^{n}\right)$, or $v_{z\left(P_{1}\right)}^{n}$ lies in $v_{x}^{n} \vec{C}_{x}^{n} v_{z\left(P_{2}\right)}^{n}$ for every pair $\left(P_{1}, P_{2}\right) \in \mathcal{P}$ (and we say that $a$ belongs between $x$ and $b$ on $C_{x}^{n}$ ).

Proof of Claim 2. Suppose the claim is false. Let $C:=C_{x}^{n}$ and $v:=v_{x}^{n}$, and choose $\left(P_{1}, P_{2}\right),\left(P_{3}, P_{4}\right) \in \mathcal{P}$ so that, with $z_{i}:=z\left(P_{i}\right)$ and $v_{i}:=v_{z_{i}}^{n}$ for $i=1, \ldots, 4$,

( $\alpha) v_{1} \in v_{2} \vec{C} v$ while $v_{3} \in v \vec{C} v_{4}$ 
$(\beta)$ subject to $(\alpha),\left|v_{1} \vec{C} v\right|+\left|v \vec{C} v_{2}\right|+\left|v \vec{C} v_{3}\right|+\left|v_{4} \vec{C} v\right|$ is minimum.

Let us show the following:

(1) If $v_{4}$ lies in $v_{1} \vec{C} v$ then $z_{1}=z_{4}$.

Suppose not and let $w$ be the first vertex of $z_{4} P_{4} b$ which also lies in $P_{2}$. If $z_{4} P_{4} w$ avoids $P_{1}$, then $z_{2} P_{2} w P_{4} z_{4}$ and $P_{1}$ are crossing $H^{n_{-}}$ paths contradicting (e). So $z_{4} P_{4} w$ has a (first) vertex $w^{\prime}$ on $P_{1}$. But now $\left(x P_{1} w^{\prime} P_{4} z_{4}, P_{2}\right),\left(P_{3}, P_{4}\right)$ would have been a better choice than $\left(P_{1}, P_{2}\right),\left(P_{3}, P_{4}\right)$.

In the same way one can show:

(2) If $v_{1}$ lies in $v_{4} \vec{C} v$ then $z_{1}=z_{4}$.

Taken together, (1) and (2) imply that $z_{1}=z_{4}$. Similarly it follows that $z_{2}=z_{3}$. Let $P$ be a $z_{1}-z_{2}$ path in $H^{n}$ and $P^{\prime}=x \cdots y$ an $x-P$ path in $H^{n}$ (Fig. 8). Note that $x \neq y$ since $d_{H}(x) \leq 3$. Since $z_{1} \neq z_{2}, y$ cannot be equal
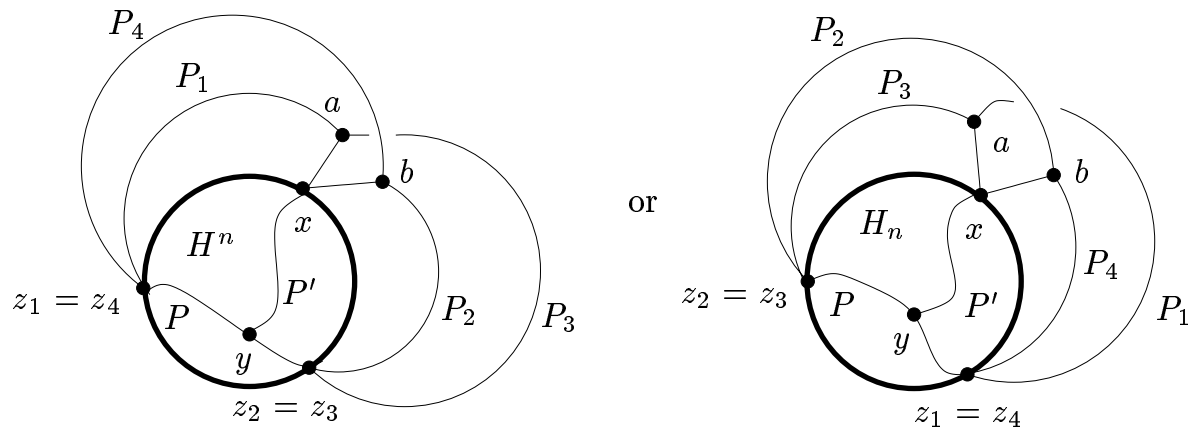

Figure 8: The proof of Claim 2

to both $z_{1}$ and $z_{2}$. We assume that $y \neq z_{2}$; the case of $y \neq z_{1}$ is analogous. Now either the cycle $z_{1} P_{4} x P^{\prime} y P z_{1}$ separates $a$ from $z_{2}=z_{3}$ or the cycle $z_{1} P_{1} x P^{\prime} y P z_{1}$ separates $b$ from $z_{2}=z_{3}$. This contradicts either the fact that $a P_{3} z_{3}$ avoids $z_{1} P_{4} x P^{\prime} y P z_{1}$ or the fact that $b P_{2} z_{2}$ avoids $z_{1} P_{1} x P^{\prime} y P z_{1}$.

Case 1: $H^{n+1}$ was obtained from $H^{n}$ by adding a new vertex a and joining it to $x \in H^{n}$.

Let $m(n+1):=m(n)+1$. For all cycles $C$ of the form $C_{z}^{n}$ let $C_{z}^{n+1}$ be the large cycle of $G_{m(n+1)}^{*}$ inside $C$. For all vertices $z \in H^{n}$ let

$$
V_{z}^{n+1}:= \begin{cases}V_{z}^{n} \cup V\left(P_{v_{z}^{n}}\right) & \text { if } d_{H^{n+1}}(z)<d_{H}(z) \\ V_{z}^{n} & \text { otherwise. }\end{cases}
$$


Suppose first that $x$ has a neighbour $b \neq a$ in $H$ (which may or may not lie in $H^{n}$ ) such that $x b$ is not an edge of $H^{n}$. Recall that $b$ is unique, so either Claim 1 or Claim 2 applies (but not both). If $a$ belongs between $x$ and $b$, let $u$ be the first (red) vertex on $\vec{C}_{x}^{n}$ after $v_{x}^{n}$. If $a$ belongs between $b$ and $x$, or if $x$ has no such neighbour $b$ in $H$, let $u$ be the first (red) vertex on $\overleftarrow{C}_{x}^{n}$ after $v_{x}^{n}$. Hence, $u$ is adjacent to $v_{x}^{n}$. Define

$$
V_{a}^{n+1}:= \begin{cases}\{u\} & \text { if } d_{H^{n+1}}(a)=d_{H}(a) \\ V\left(P_{u}\right) & \text { otherwise }\end{cases}
$$

and for each $z \in H-H^{n+1}$ let

$$
V_{z}^{n+1}:=\emptyset
$$

It remains to check that the sets $V_{z}^{n+1}$ satisfy (a)-(e) for all $z \in H$. The conditions (a)-(d) are straightforward. For (e) note that for all $z \in H^{n}$ the cyclic order of the vertices $v_{z}^{n+1}$ on any given cycle $C_{y}^{n+1} \in \mathcal{C}_{m(n+1)}$ agrees with the cyclic order of the corresponding vertices $v_{z}^{n}$ on $C_{y}^{n}$. This together with the validity of (e) for $n$ implies that crossing paths $P, P^{\prime}$ must be such that $P$ (say) ends in a (otherwise $P, P^{\prime}$ cross also for $n$ ) and $P^{\prime}$ ends in $x$ (otherwise $P^{\prime}$ crosses $P a x$ for $n$ ). Let $P=: a \cdots z$ and $P^{\prime}=: x b \cdots z^{\prime}$ (where possibly $b=z^{\prime}$ ). If $a$ belongs between $x$ and $b$ then both $v_{a}^{n+1}, v_{z}^{n+1}$ lie in $v_{x}^{n+1} \vec{C}_{x}^{n+1} v_{z^{\prime}}^{n+1}$ (irrespective of whether $b \in H^{n}$ ), i.e. $P$ and $P^{\prime}$ do not cross. If not, then $a$ belongs between $b$ and $x$, so both $v_{a}^{n+1}, v_{z}^{n+1}$ lie in $v_{z^{\prime}}^{n+1} \vec{C}_{x}^{n+1} v_{x}^{n+1}$, and again $P$ and $P^{\prime}$ do not cross.

Case 2. $H^{n+1}$ was obtained from $H^{n}$ by adding an edge $x y$, for some vertices $x, y$ of $H^{n}$.

By (d), $V_{x}^{n}$ and $V_{y}^{n}$ meet the same cycle of $\mathcal{C}_{m(n)}$, i.e. $C_{x}^{n}=C_{y}^{n}=: C$. Let $s_{0}$ be the red neighbour of $v_{x}^{n}$ in $v_{x}^{n} \vec{C} v_{y}^{n}$ and $t_{0}$ the red neighbour of $v_{y}^{n}$ in $v_{y}^{n} \vec{C} v_{x}^{n}$. Let $s_{1}, \ldots, s_{k}$ be the inner vertices of $v_{x}^{n} \vec{C} v_{y}^{n}$ of the form $v_{z}^{n}$ (in order). Similarly, let $t_{1}, \ldots, t_{l}$ be the inner vertices of $v_{y}^{n} \vec{C} v_{x}^{n}$ of the form $v_{z}^{n}$ (in order). Let $s_{k+1}$ be the red neighbour of $v_{y}^{n}$ in $v_{x}^{n} \vec{C} v_{y}^{n}$ and $t_{l+1}$ the red neighbour of $v_{x}^{n}$ in $v_{y}^{n} \vec{C} v_{x}^{n}$.

Now let

$$
m(n+1):=\max \{m(n)+k+2, m(n)+l+2\}
$$

and

$$
N:=m(n+1)-m(n)
$$


For all vertices $z \in H^{n}$ such that $V_{z}^{n}$ does not meet $C$, extend $V_{z}^{n}$ by setting

$$
V_{z}^{n+1}:= \begin{cases}V_{z}^{n} \cup V\left(P_{v_{z}^{n}}^{N}\right) & \text { if } d_{H^{n+1}}(z)<d_{H}(z) \\ V_{z}^{n} & \text { otherwise. }\end{cases}
$$

Note that $k+1 \leq n \leq m(n) \leq 2^{m(n)+2}-2$ by (c). Thus we may apply Lemma 2 to obtain disjoint $P_{s_{0}}-P_{s_{k+1}}$ paths $P_{1}, \ldots, P_{k+1}$. Let $P_{0}:=s_{0}$. For each $i=0, \ldots, k+1$, let $R\left(s_{i}\right)$ be the path through $f\left(C_{v_{x}^{n} v_{y}^{n}}\right)$ that starts at $s_{i}$, follows $P_{s_{i}}$ until it hits $P_{i}$, then follows $P_{i}$ to $P_{s_{0}}$, then traverses the unique edge $p q$ from its vertex $p$ on $P_{s_{0}}$ to the small cycle of $G_{m(n)+1}^{*}$ inside $C$ preceding $s_{0}$, and finishes with the path $P_{q}^{N-1}$ to end on, say, $C_{s_{0}}^{\prime} \in \mathcal{C}_{m(n+1)}$ (Fig. 9). Similarly define $C_{t_{0}}^{\prime}$ and, for all $i=0, \ldots, l+1$, disjoint $t_{i^{-}} C_{t_{0}}^{\prime}$

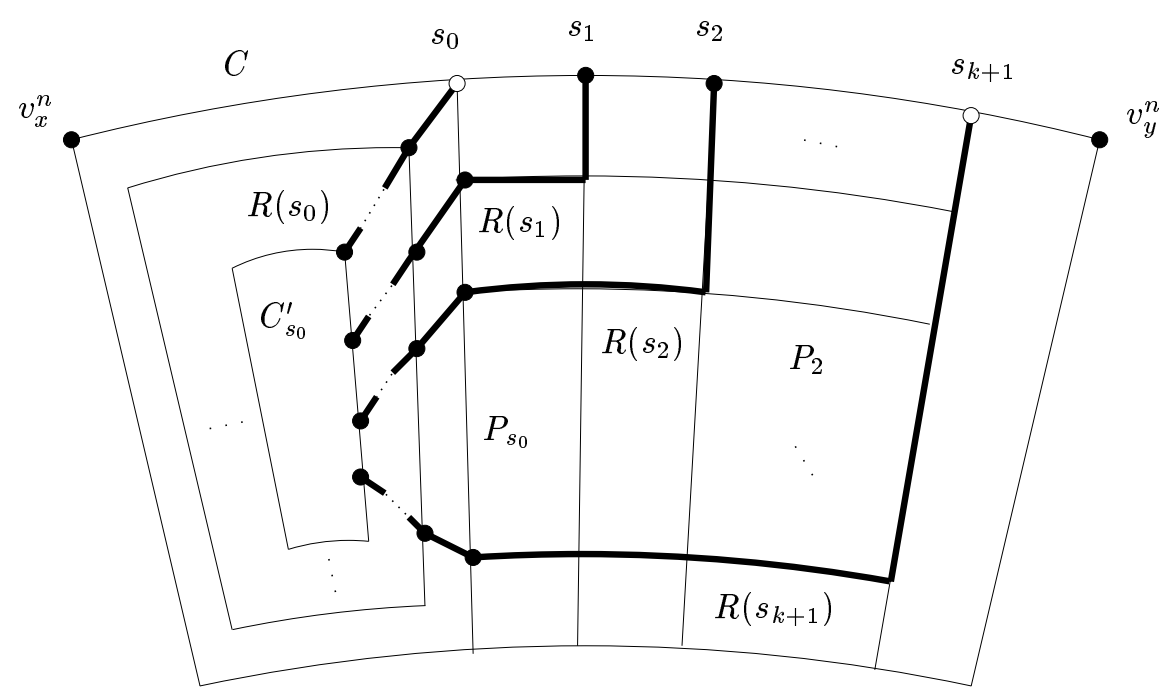

Figure 9: Joining the $s_{i}$ to $C_{s_{0}}^{\prime}$ by paths $R\left(s_{i}\right)$

paths $R\left(t_{i}\right)$ through $f\left(C_{v_{y}^{n} v_{x}^{n}}\right)$.

For all vertices $z \in H^{n}$ such that $z \neq x, y$ and $V_{z}^{n}$ meets $C$, define

$$
V_{z}^{n+1}:=V_{z}^{n} \cup V\left(R\left(v_{z}^{n}\right)\right) .
$$

Note that if $d_{H^{n+1}}(x)<d_{H}(x)$, then $H$ has a unique vertex $y^{\prime}$ such that $x$ is adjacent to $y^{\prime}$ in $H$ but not in $H^{n+1}$. Then $y^{\prime} \in H^{n}$ by condition (iii) on the choice of the sequence $\left(H^{n}\right)_{n=0}^{\infty}$, and $v_{y^{\prime}}^{n} \in C$ by (d). Extend $V_{x}^{n}$ by 
setting

$$
V_{x}^{n+1}:= \begin{cases}V_{x}^{n} \cup V\left(P_{v_{x}^{n}}\right) & \text { if } d_{H^{n+1}}(x)=d_{H}(x) \\ V_{x}^{n} \cup V\left(P_{v_{x}^{n}} \cup R\left(s_{0}\right)\right) & \text { if } d_{H^{n+1}}(x)<d_{H}(x) \text { and } v_{y^{\prime}}^{n} \in\left\{s_{1}, \ldots, s_{k}\right\} \\ V_{x}^{n} \cup V\left(P_{v_{x}^{n}} \cup R\left(t_{l+1}\right)\right) & \text { if } d_{H^{n+1}}(x)<d_{H}(x) \text { and } v_{y^{\prime}}^{n} \in\left\{t_{1}, \ldots, t_{l}\right\} .\end{cases}
$$

Let $C^{\prime}$ be the large cycle of $G_{m(n)+1}^{*}$ inside $C$, and let $W$ denote the set of inner vertices of the subpath of $C^{\prime}$ between the endpoints of $P_{v_{y}^{n}}$ and $P_{v_{x}^{n}}$ that follows the clockwise orientation of $C^{\prime}$. As before denote by $x^{\prime}$ the vertex adjacent to $y$ in $H$ but not in $H^{n+1}$, if it exists. Define

$$
V_{y}^{n+1}:= \begin{cases}V_{y}^{n} \cup W \cup V\left(P_{v_{y}^{n}}\right) & \text { if } d_{H^{n+1}}(y)=d_{H}(y) \\ V_{y}^{n} \cup W \cup V\left(P_{v_{y}^{n}} \cup R\left(t_{0}\right)\right) & \text { if } d_{H^{n+1}}(y)<d_{H}(y) \text { and } v_{x^{\prime}}^{n} \in\left\{t_{1}, \ldots, t_{l}\right\} \\ V_{y}^{n} \cup W \cup V\left(P_{v_{y}^{n}} \cup R\left(s_{k+1}\right)\right) & \text { if } d_{H^{n+1}}(y)<d_{H}(y) \text { and } v_{x^{\prime}}^{n} \in\left\{s_{1}, \ldots, s_{k}\right\}\end{cases}
$$

(Fig. 10.) For all vertices $z \in H-H^{n+1}$ let $V_{z}^{n+1}:=\emptyset$. Again, the sets $V_{z}^{n+1}$

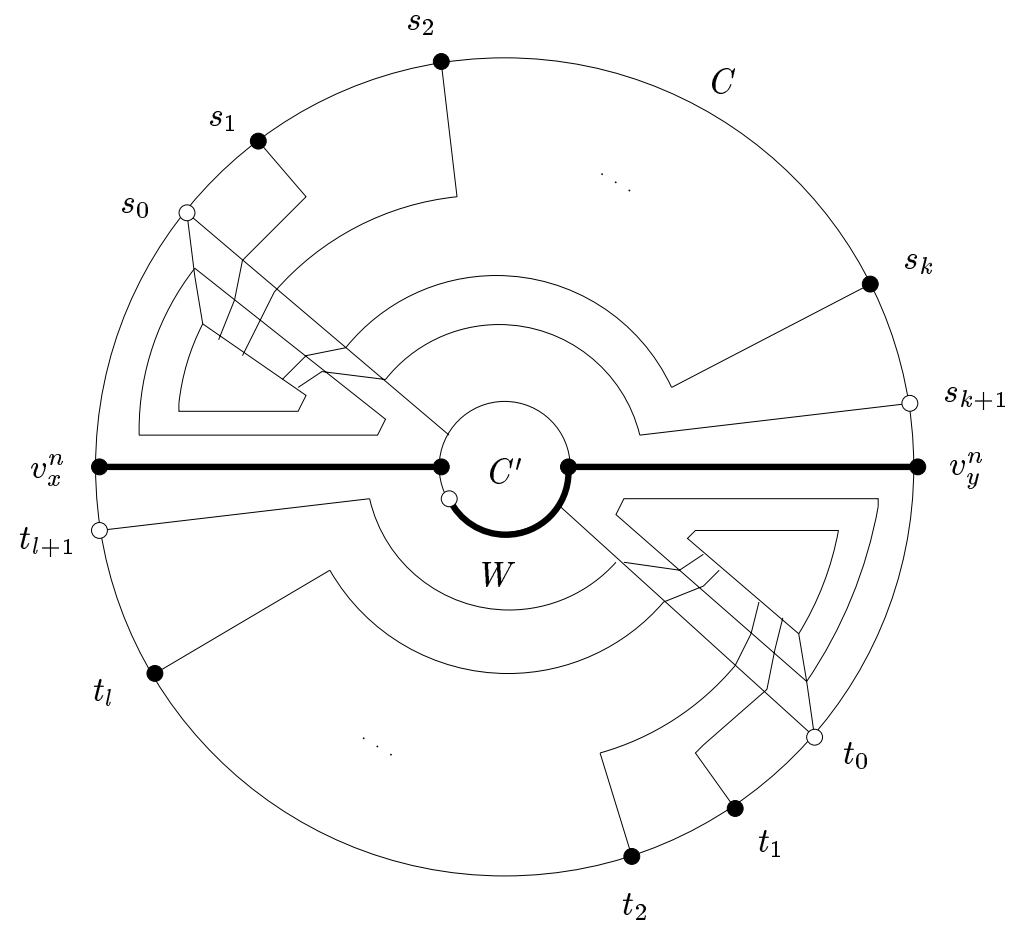

Figure 10: Accommodating the edge $x y$ 
satisfy (a)-(e). Indeed, checking the conditions (a)-(c) is straightforward. Any counterexample to (d) would be an $H^{n}$-path crossing $x y$, contradicting (e) for $n$. Finally, (e) for $n+1$ follows from (e) for $n$ since the cyclic order of the vertices $v_{z}^{n+1}$ on $C_{s_{0}}^{\prime}$ and on $C_{t_{0}}^{\prime}$ reflects that of the corresponding vertices $v_{z}^{n}$ on $C$ (and similarly for the other cycles in $\mathcal{C}_{m(n+1)}$ ).

For all vertices $x \in H$ let $V_{x}:=\bigcup_{n=0}^{\infty} V_{x}^{n}$. By (a), these sets $V_{x}$ inherit connectedness in $G^{*}$ from the sets $V_{x}^{n}$. Hence by (b), $H$ is a minor of $G^{*}$ with branch sets $V_{x}$, for all $x \in H$.

\section{Open Problems}

As the construction of our universal graph $G^{*}$ shows at once, $G^{*}$ is locally finite; indeed $\Delta\left(G^{*}\right)=8$. Thus if $G$ is a planar graph with a vertex $x$ of infinite degree, the branch set of $x$ in any embedding of $G$ in $G^{*}$ as a minor will be infinite. One obvious question that we have not addressed is whether this can be avoided:

Problem 5 Is there a planar graph $G^{*}$ such that every planar graph can be embedded in $G^{*}$ as a minor with finite branch sets?

Another obvious strengthening of Theorem 4 would be to ask for a universal planar graph with respect to the topological minor relation:

Problem 6 Is there a planar graph $G^{*}$ that contains a subdivision of every planar graph as a subgraph?

Abstracting from planarity, one might ask which other minor-closed graph properties have a (countable) universal graph. For example:

Problem 7 For which graphs $X$ is there a countable graph $G^{*}$ without an $X$ minor such that every countable graph without an $X$ minor is a minor of $G^{*}$ ?

For the complete graphs $X=K^{3}$ and $X=K^{4}$, there are graphs without an $X$ minor that are universal for this property even with respect to the subgraph relation. Indeed, the $\aleph_{0}$-regular tree contains every countable graph without a $K^{3}$ minor as a subgraph, and a universal graph for $K^{4}$ can be obtained by recursively pasting triangles together along edges (see [1, Prop. 8.3.1]). For $n \geq 5$, there is no (countable) subgraph-universal graph without a $K^{n}$ minor [2], but we do not know whether these classes 
have minor-universal elements. By a result of Halin [4], the edge-maximal graphs without a $K^{5}$ minor are precisely those that have a certain treedecomposition into countable maximal planar graphs and copies of the Wagner graph $W$ (see [1]); thus, Theorem 4 may help in the construction of any minor-universal graph for $X=K^{5}$. Similarly, the graphs without a $K^{\aleph_{0}}$ minor have a characterization by their tree-decompositions that involves planar graphs [3], so here too Theorem 4 might conceivably be of help.

Interestingly, the sphere stands out in that for no other closed surface $S$ does the class of graphs embeddable in $S$ have a minor-universal element (C. Thomassen, personal communication). Indeed, any such graph $G^{*}$ would contain a cycle $C^{*}$ whose deletion reduces the Euler genus of $G^{*}$. Then every minor of $G^{*}$ can be embedded in a smaller surface than $S$ after the deletion of at most $\left|C^{*}\right|$ vertices. This, however, will not be the case for every graph embeddable in $S$.

\section{References}

[1] R. Diestel, Graph Theory, Graduate Texts in Mathematics 173, Springer-Verlag 1997.

[2] R. Diestel, R. Halin and W. Vogler, Some remarks on universal graphs, Combinatorica 5 (4) (1985), 283-293.

[3] R. Diestel and R. Thomas, Excluding a countable clique, submitted.

[4] R. Halin, Ein Zerlegungssatz für unendliche Graphen und seine Anwendung auf Homomorphiebasen, Math. Nachr. 33 (1967), 91-105.

[5] P. Komjáth and J. Pach, Universal elements and the complexity of certain classes of infinite graphs, Discrete Math. 95 (1991), 255-270.

[6] D. Kühn, A characterization of the minors of the infinite grid, in preparation.

[7] J. Pach, A problem of Ulam on planar graphs, European J. Combin. 2 (1981), 357-361.

Reinhard Diestel and Daniela Kühn,

Faculty of Mathematics

TU Chemnitz

09107 Chemnitz

Germany 